\title{
Une gloire singulière. Pour une meilleure réception de Rimbaud au Japon
}

A singular glory. For a better reception of Rimbaud in Japan

\section{Yoshikazu Nakaji}

\section{(2) OpenEdition}

1 Journals

\section{Édition électronique}

URL : https://journals.openedition.org/rief/7313

DOI : $10.4000 /$ rief.7313

ISSN : 2240-7456

\section{Éditeur}

Seminario di filologia francese

\section{Référence électronique}

Yoshikazu Nakaji, « Une gloire singulière. Pour une meilleure réception de Rimbaud au Japon », Revue italienne d'études françaises [En ligne], 11 | 2021, mis en ligne le 15 novembre 2021, consulté le 18 novembre 2021. URL : http://journals.openedition.org/rief/7313 ; DOI : https://doi.org/10.4000/rief. 7313

Ce document a été généré automatiquement le 18 novembre 2021.

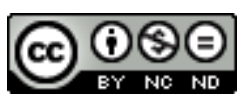

Les contenus de la RIEF sont mis à disposition selon les termes de la Licence Creative Commons Attribution - Pas d'Utilisation Commerciale - Pas de Modification 4.0 International. 


\title{
Une gloire singulière. Pour une meilleure réception de Rimbaud au Japon
}

\author{
A singular glory. For a better reception of Rimbaud in Japan
}

Yoshikazu Nakaji

\section{Au commencement était le rythme figé}

1 L'introduction de la poésie occidentale commence au Japon vers le milieu du XIX siècle, quand, sous la pression des puissances occidentales, le pays se décide enfin à s'ouvrir à l'extérieur après un isolement quasi total de deux siècles et demi. L'assimilation de tout ce qui relève de la culture occidentale passe par la langue. L'apprentissage des langues européennes devient une nécessité imminente. Le Japon est un des rares pays d'Asie à avoir échappé à la colonisation linguistique comme à la colonisation tout court. Les Japonais ont eu l'habitude de tout intégrer dans leur culture par la traduction. La littérature ne fait pas exception.

2 Pendant longtemps depuis l'antiquité, la poésie japonaise connut deux courants distincts: la poésie chinoise, d'un côté, que les Japonais ont pratiquée en la réinterprétant selon la grammaire japonaise et, d'un autre côté, les formes brèves proprement japonaises, le waka (le chant japonais) et plus tard le haiku qui en dérive, lesquelles sont basées sur un rythme familier à leur oreille, l'alternance de 7 et 5 syllabes. En 1882 paraît une première anthologie de la poésie occidentale, Shintaishish $\hat{o}^{1}$ (Recueil de poèmes dans le style nouveau). Ouvrage mixte composé de quatorze poèmes anglo-américains et de cinq créations, le recueil comportait non seulement des poèmes de Tennyson, de Gray ou de Longfellow mais aussi des extraits de Hamlet (« To be or not to be... »), et même un extrait de Principes de sociologie de Herbert Spencer, sociologue évolutionniste alors à la mode. Vers ou prose, poème ou essai, traduction ou création, on mettait tout dans le moule de 7 et 5 syllabes, en prétendant que c'étaient là des "poèmes dans le style nouveau». La nouveauté du recueil résidait, pour les trois 
compilateurs-traducteurs, dans son dépassement des formes brèves malgré le maintien de la rythmique fondamentale qui les fondait.

Pour les lecteurs d'aujourd'hui comme pour ceux de l'époque, ces essais de traduction et de création sont mécaniques et sans inspiration, ridicules par leur formalisme creux. Mais c'était là le premier pas d'une langue poétique en état de renaissance, qui devait continuer désormais à rechercher une forme et une musique toujours mieux appropriées. Cependant, la prégnance de la cadence 7-5 était si forte que les tentatives de baser toute poésie sur la cadence traditionnelle devaient se poursuivre jusqu'au début du XXe siècle, parallèlement aux efforts pour l'adoucir en la mêlant avec d'autres types de rythme.

4 Ainsi, la traduction en langue japonaise de la poésie occidentale soulève la question de la rénovation de la langue poétique. Les poètes partagent cette nécessité avec les traducteurs, car la poésie moderne elle-même devait s'inventer une forme et une musique qui se démarquent de celles du waka et du haiku. L'exigence rimbaldienne de

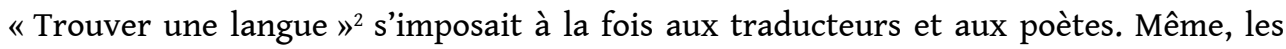
travaux de certains traducteurs et leurs anthologies de poèmes traduits suggèreront aux poètes une voie à emprunter.

\section{Une curieuse absence}

Kaichôon (Le Bruit de la marée, 1905) réalisée par Bin Ueda (1874-1916), Sango-shû (Corail, 1913) par le romancier Kafû Nagai (1879-1959) et Gekka no ichigun (Un troupeau sous la lune, 1925) par Daigaku Horiguchi (1892-1981), telles sont les trois premières anthologies $^{3}$ qui ont contribué à faire connaître aux lecteurs japonais la poésie française moderne, car celle-ci avait pris du retard par rapport à la poésie angloallemande.

6 La première anthologie comporte des poèmes anglais, allemands, italiens et provençaux (les deux derniers sont retraduits à partir de leurs traductions anglaises) mais la majorité des poèmes recueillis (31 sur 57) sont des poèmes français (Baudelaire, Mallarmé, Verlaine, des Parnassiens et des symbolistes fin de siècle) et belges (Verhaeren et Rodenbach). Tout en s'appuyant sur l'alternance traditionnelle de 7 et 5 syllabes, le travail de Bin Ueda dépasse de loin le formalisme vide du Shintaishishô. Au moyen d'un vocabulaire élégant et somptueux puisé dans le japonais ancien et dans le chinois classique, le traducteur excelle à traduire les alexandrins réguliers comme ceux de Leconte de Lisle (Midi) dans une cadence japonaise non moins régulière. Il est aussi à l'aise avec les vers brefs et légers comme Chanson d'automne de Verlaine qu'il traduit en vers de 5 syllabes volontairement monotones et qui reste l'une des pièces les plus célèbres de son anthologie. Son travail constitua un exemple du Bungo teikeishi (vers fixe en langue littéraire), prospère à l'époque. Rimbaud est absent de cette anthologie ${ }^{4}$. Mais, à la mort prématurée du traducteur on découvrit parmi ses manuscrits quatre brouillons de traduction du Bateau ivre dont l'un est presque achevé : une seule strophe $\mathrm{y}$ manque. Malgré son intérêt profond pour ce grand poème de Rimbaud, Ueda n'arriva jamais à en réaliser une traduction qui le satisfasse. En effet, les alexandrins de Rimbaud, pleins d'enjambements et marqués par le peu de respect de la césure au milieu du vers, voire par une syntaxe volontairement difforme, sont loin de la sensibilité poético-linguistique du traducteur qui convenait au contraire parfaitement à la versification classique d'un Leconte de Lisle. 
7 La deuxième anthologie est entièrement consacrée à la poésie française. Elle contient 41 poèmes modernes, dont Baudelaire ( 7 poèmes), Verlaine ( 7 poèmes) et Henri de Régnier (10 poèmes). Le seul poème recueilli de Rimbaud est Sensation, un petit poème en deux quatrains d'alexandrins réguliers où n'apparaît encore ni l'ironie ni le sarcasme qui lui sont propres. Le titre japonais choisi par Kafû Nagai est Sozoroaruki (Flânerie). Tout en utilisant la langue écrite, le traducteur libère ses vers du rythme figé 7-5 pour rejoindre le mouvement du bungo jiyûshi (vers libre en langue littéraire) que pratiquaient alors les poètes qui se qualifiaient eux-mêmes de «symbolistes ». Pour Sensation le traducteur se permet davantage de licence : il traduit le premier quatrain en six et le second en sept vers libres. Du coup, la seule traduction de Rimbaud par Kafû Nagai est plutôt une adaptation, mais c'est là une belle réussite qui préserve la fraîcheur du texte original dans une musique neuve.

8 La troisième anthologie est un recueil important, contenant 339 poèmes de 66 poètes français. Y figurent, d'une part, les grands habitués du XIX ${ }^{\mathrm{e}}$ siècle, Baudelaire, Mallarmé, Verlaine et même Charles Cros et, d'autre part, les décadents ou symbolistes fin de siècle, Laforgue, Samain, Henri de Régnier, Gourmont. Mais la place majeure est réservée aux poètes du $\mathrm{XX}^{\mathrm{e}}$ siècle, Apollinaire, Jacob, Cocteau, Radiguet, ainsi que Francis Jamme et Paul Fort. Le traducteur avouera plus tard à propos de son anthologie que chaque fois qu'un poème lui plaisait, il voulut le traduire " pour avoir l'illusion de mieux le posséder $»^{5}$. En langue japonaise, les poèmes recueillis ici revêtent une vie nouvelle comme s'il s'agissait des propres créations de Daigaku Horiguchi. Le traducteur inaugurait de fait le kôgo jiyûshi (vers libre en langue courante), modalité qui devait désormais constituer la base de la pratique poétique et reste encore en vigueur de nos jours. Curieusement, Rimbaud ne figure pas non plus dans cette anthologie. Horiguchi s'explique ainsi sur cette absence :

Il y a ce genre de poètes que j'adore mais n'arrive d'aucune façon à assimiler dans mon propre japonais: est-ce à cause de nos respirations différentes? Depuis longtemps Rimbaud et Mallarmé me font pousser de tels soupirs. C'est pourquoi dans Gekka no ichigun ne figure pas un seul poème de Rimbaud, même parmi les plus courts. Il m'aura fallu plus de dix ans après Gekka no ichigun, pour réussir enfin à mener à bien ma traduction du Bateau ivre. ${ }^{6}$

9 Ainsi, les traducteurs qui jouèrent un rôle capital dans la diffusion de la poésie française et servirent en même temps de modèle à la création poétique de leur temps, semblent avoir été tous mal à l'aise avec le vers de Rimbaud.

\section{Hidéo Kobayashi (1902-1983), traducteur d'Une saison en enfer}

10 C'est dans les années 1930 que commence véritablement l'introduction de Rimbaud au Japon. Les proses de Rimbaud, Une saison en enfer et les Illuminations, furent traduites pour la première fois en 1930 par Hidéo Kobayashi, futur fondateur de la critique moderne au Japon.

11 L'étude intensive du symbolisme français avait commencé dès les années 1920 à l'Université impériale de Tokyo. Étudiant alors inscrit à cette université, Kobayashi s'intéresse à la littérature moderne de Baudelaire aux écrivains de la NRF. Il est surtout attiré par Rimbaud et Valéry. Lycéen, il avait été initié au symbolisme français par l'ouvrage Symbolist Movement in Literature d'Arthur Symons, traduit en japonais en 1913. 
12 Kobayashi a laissé trois essais sur Rimbaud qui datent respectivement de 1926, 1930 et $1947^{7}$. Ceux-ci illustrent bien sa posture critique. Le premier en particulier, initialement intitulé Arthur Rimbaud, le trancheur de la vie, témoigne de son approche intuitive et de son ton catégorique. Le titre renvoie à la façon de vivre de l'auteur, sa résistance à la civilisation occidentale, sa mue perpétuelle : «Lorsque, chez un artiste, l'intelligence surpasse la sensibilité, il tranche la vie en morceaux. [...] [Rimbaud] défia la civilisation par toutes sortes de métamorphose $»^{8}$.

13 Son approche critique consiste à s'attacher exclusivement au "moi créateur » des grands artistes, au face-à-face du génie avec sa «fatalité ». La « basse dominante de la fatalité $\|^{9}$ : c'est à Valéry qu'il emprunte cette métaphore musicale qui revient fréquemment dans ses premiers écrits. Traducteur quelques années plus tard de La Soirée avec Monsieur Teste, Kobayashi critique avait pour méthode fondamentale de se confronter aux grands esprits créateurs pour capter «intuitivement " leur drame intérieur, à l'instar de Valéry face à Léonard de Vinci.

14 Kobayashi écrira plus tard sur d'autres génies comme Mozart, Dostoïevski, Van Gogh ou les grands classiques japonais. Chez lui, le génie comme fatalité n'a rien de transcendantale. Le critique vise à capter la personnalité intense propre à chaque génie, sa façon d'être fondamentale. Cet attachement aux génies va toujours de pair avec l'identification et le partage, à tel point qu'on ne distingue plus très bien s'il parle des autres ou de lui-même. «La critique consiste à parler de soi en utilisant les œuvres des autres comme prétexte $»^{10}$. En effet, dans sa critique, l'accent est mis davantage sur l'effet produit par cet objet sur lui que sur l'objet lui-même. Voici un fameux passage de son troisième essai sur Rimbaud où l'auteur rapporte sa rencontre avec Rimbaud et Une saison en enfer :

C'est au printemps de ma vingt-troisième année que j'ai rencontré Rimbaud pour la première fois. Je me promenais dans le quartier de Kanda, pourrais-je écrire ainsi si l'on veut, lorsqu'un homme inconnu s'avançant vers moi, m'a tout d'un coup mis à terre. Je n'y étais pas prêt. Je n'avais point imaginé de quel explosif était piégé ce petit volume d'aspect misérable d'Une saison en enfer, édition du Mercure de France. De plus, son appareil d'allumage était tellement sensible que la faiblesse de mes compétences linguistiques n'y était pour rien. Le petit explosif a magnifiquement éclaté ; dès lors, pendant plusieurs années, je suis demeuré au sein d'un événement nommé Rimbaud. ${ }^{11}$

15 Or, Une saison en enfer par Kobayashi témoigne de cette identification sur le plan de la traduction aussi. Le traducteur devient en l'occurrence un excellent acteur sachant incarner son rôle: il réussit à recréer en japonais la parole du narrateur rimbaldien s'adressant constamment à un interlocuteur dont l'identité demeure incertaine. Ce monologue dialogique ou dialogue en puissance est modulé sur toute une gamme de pensées et de sentiments. La traduction de Kobayashi épouse cette oralité impétueuse caractéristique de l'auteur. Le nom de Rimbaud reste inséparable de celui de Hidéo Kobayashi et de sa traduction d'Une saison en enfer intitulée : Jigokuno kisetsu.

Mais la traduction de Kobayashi est problématique. Prenons comme exemple l'une des phrases-clefs du prologue : «Et j'ai joué de bons tours à la folie ${ }^{12}$. Elle définit la série de révoltes du héros-narrateur comme une approche progressive de la «folie » (allégorisée ici sans majuscule) et annonce en même temps le diptyque central surtitré Délires. Rimbaud veut dire ceci : la folie l'a effleuré plus d'une fois dans sa tentative existentielle et artistique mais chaque fois il a réussi à l'esquiver de justesse. Or, Kobayashi a traduit cette phrase comme ceci: "shikamo orega enjitamonowa 
sokonukeno gozakyôdatta ", soit " et j'ai joué des plaisanteries sans bornes ». Il n'a pas vu la construction "jouer de bons tours à... ", et a pris "à la folie " pour une locution adjectivale d'emphase et non pour complément d'objet indirect du verbe "jouer ». Le narrateur semble se dépeindre comme un simple farceur. Du coup, un aspect majeur de l'œuvre, ce qu'on peut appeler la « poétique de la folie » chez Rimbaud ${ }^{13}$ est estompé.

Voici maintenant les fameuses paroles de l'Époux infernal citées par la Vierge folle :

Je n'aime pas les femmes, L'amour est à réinventer, on le sait. Elles ne peuvent que vouloir une position assurée. La position gagnée, cœur et beauté sont mis de côté $[\ldots]^{14}$

Voici la traduction de Kobayashi, que je retraduis en français :

Je n'aime pas les femmes. L'amour est un maquillage, on le sait. Elles ne peuvent que vouloir s'assurer une position. La position assurée, cœur et beauté sont mis de côté [...])

Telle que Kobayashi la comprend, la proposition «L'amour est à réinventer » dit que les femmes feignent d'être aimables pour obtenir le statut d'épouse : ce sont elles qui « réinventent », c'est-à-dire "forgent» l'apparence. Mais dans le texte original, c'est l'Époux infernal qui ressent la nécessité de recréer le vrai amour qui n'existe pas, étant donné le comportement des femmes qui se font calculatrices pour sortir de leur misère.

Le célèbre "opéra fabuleux $»^{15}$ dans Alchimie du verbe devient dans la traduction de Kobayashi un "opéra fictif» («kakuuno opera»). C'est là un contresens grave et déplorable, car l'« opéra» en question désigne un état d'âme dans lequel le poète entend successivement plusieurs voix "angéliques", qui pourtant "s'expliquent vertement $»^{16}$. Tout se passe comme s'il s'était lui-même transformé en un espace opéradique intérieur où se croisent ces voix appelées «multiples sœurs ». L'« opéra fabuleux » n'est pas du tout fictif: il est "extraordinaire, à peine croyable bien que réel » (Littré).

Dans l'histoire de la réception de Rimbaud, la contribution de Hideo Kobayashi est immense. Malgré tous ces défauts, elle a longtemps bénéficié d'un accueil enthousiaste. Une saison en enfer par Kobayashi a fait entrer Rimbaud dans la culture japonaise, en lui réservant une place privilégiée parmi les poètes non seulement français mais occidentaux. En retour, Rimbaud, à travers cette traduction et les trois essais que Kobayashi lui a consacrés, a contribué à rendre célèbre le nom de son traducteur et critique.

\section{Chûya Nakahara (1907-1937), traducteur des vers de Rimbaud}

Le travail du poète Chûya Nakahara est tout aussi important. Cadet de Kobayashi de cinq ans, quasiment autodidacte en langue française, par ailleurs rival malheureux de ce dernier dans un triangle amoureux, Nakahara est attaché aux vers de Rimbaud comme pour délimiter son propre domaine par rapport à son aîné. Mais c'est peut-être aussi parce que le vers convenait mieux à son talent de poète. L'invention rythmique dont il fait preuve dans sa traduction apporte une réponse aux problèmes laissés par les trois premiers anthologistes, tous désarçonnés par les vers de Rimbaud.

Depuis ses origines, la poésie japonaise ne recourt ni au mètre (au sens occidental du terme) ni à la rime, sauf dans de rares essais dans la modernité qui se révélèrent vite stériles ${ }^{17}$. À cela on peut supposer plusieurs raisons, qui sont toutes liées à la structure 
de la langue japonaise. La plus importante est qu'il n'y a en japonais que cinq voyelles ([a], [i], [u], [e], [o]); chaque son japonais est la combinaison d'une consonne et d'une voyelle (à l'exception de ces voyelles mêmes qui constituent chacune un son). Le système phonétique du japonais est bien plus simple que celui du français. Il est comme un tissu dans lequel chaîne et trame sont tissés uniformément, une surface plane sans bosses de voyelles nasales sonores ni plis serrés de consonnes consécutives, comme le montrera plus loin la transcription phonétique de la traduction japonaise d'un extrait des Poètes de sept ans.

Dans les années 1920 et 1930, la cadence traditionnelle de 7-5 avait perdu sa fraîcheur. Mais elle n'avait pas totalement disparu de la sphère poétique : elle hantait toujours les Japonais sensibles à la musique des mots. Un substitut efficace, un rythme nouveau indispensable à la poésie, restait à trouver. C'est dans cet « interrègne » rythmique que Nakahara fut actif comme poète et traducteur. La langue poétique japonaise mettait alors le cap sur le « vers libre ».

Sakutarô Hagiwara (1886-1942), poète fondateur du vers libre, et qui l'a lui-même perfectionné, explique les possibilités et les dangers du vers libre, dans son essai Sur le rythme du vers libre (1923). Il mentionne le "rythme intime de notre cœur, soit l'inner rhythm du poète du vers libre ». Selon lui, le poète doit abandonner les cadences toutes faites pour « accorder le rythme intime du cœur avec le rythme extérieur des mots ». Il considère le vers libre comme "l'expression suprême ", mais est vigilant face au piège de sa facilité. Ni poésie fondée sur une métrique toute faite, ni poésie en prose ne reposant sur aucun mètre, le vers libre, selon lui, a besoin malgré tout d'un rythme. Or, ce rythme est à inventer chaque fois et sa valeur n'est pas assurée d'avance.

Dans le cas du poème à cadence fixe, le rythme codifié permet, même s'il est médiocre, de donner au lecteur une impression rythmique plus ou moins agréable [...] Par contre, le vers libre de mauvaise qualité n'a aucun charme rythmique, il est perçu comme de la pure prose. [...] le vers libre raté n'a essentiellement rien de la poésie. $^{18}$

Nakahara, lui, ne visait pas tout à fait le vers libre. Afin de diversifier la musique de son vers, il en atténuait simplement la dominance de la cadence par l'usage conjoint d'autres cadences, mais il ne chassait pas la cadence traditionnelle de sa création ni de sa traduction. Prenons un passage des Poètes de sept ans (v. 22-30) et la traduction japonaise qu'il en donne :

Pitié ! Ces enfants seuls étaient ses familiers

Qui, chétifs, fronts nus, œil déteignant sur la joue,

Cachant de maigres doigts jaunes et noirs de boue

Sous des habits puant la foire et tout vieillots,

Conversaient avec la douceur des idiots !

Et si, l'ayant surpris à des pitiés immondes,

Sa mère s'effrayait ; les tendresses, profondes,

De l'enfant se jetaient sur cet étonnement.

C'était bon. Elle avait le bleu regard,

$$
\text { - qui ment }{ }^{19} \text { ! }
$$

いたはしや！彼の仲間ときた日には、

帽子もかぶらず色褪せた眼をした哀れな奴ばかり、

市場とばかりじじむさい匂ひを放げる着物の下に

泥に污れて黄や黒の、瘦せた指をば押し匿し、

言葉を交すその時は、白痴のやうにやさしい奴等、

この情けない有様を、偶々見付けた母親は

慄へ上がつて怒気含む、するとその子のやさしさは 
その母親の驚愕に、とまれかくまれ身を投げる。

母親だって嘘つきな、碧い眼をしてみるでは

ないか! 20

Voici la transcription phonétique de cette traduction japonaise, avec décompte du nombre de sons dans les segments constitutifs de chacun des 9 vers :

I-ta-wa-shi-ya !/ka-re-no-na-ka-ma-to/ki-ta-hi-ni-wa

$$
5 / 7 / 5
$$

Bo-o-shi-mo-ka-bu-ra-zu/i-ro-a-se-ta-me-wo-shi-ta/a-wa-re-na-ya-tsu-ba-ka-ri $8 / 9 / 9$

I-chi-ba-to-ba-ka-ri/ji-ji-mu-sa-i/ni-o-i-wo-a-ge-ru/ki-mo-no-no-shi-ta-ni $7 / 5 / 7 / 7$

Do-ro-ni-yo-go-re-te/ki-ya-ku-ro-no,/ya-se-ta-yu-bi-wo-ba/o-shi-ka-ku-shi, $7 / 5 / 7 / 5$

Ko-to-ba-wo-ka-wa-su/so-no-to-ki-wa,/ha-ku-chi-no-yo-o-ni/ya-sa-shi-i-ya-tsu-ra. 7/5/7/7

Ko-no-na-sa-ke-na-i/a-ri-sa-ma-wo,/ta-ma-ta-ma-mi-tsu-ke-ta/ha-ha-o-ya-wa $7 / 5 / 8 / 5$

Hu-ru-e-a-ga-'-te/do-ki-hu-ku-mu,/su-ru-to-ko-no-ko-no/ya-sa-shi-sa-wa $7 / 5 / 7 / 5$

So-no-ha-ha-o-ya-no/kyo-o-ga-ku-ni,/to-ma-re-ka-ku-ma-re/mi-wo-na-ge-ru $7 / 5 / 7 / 5$

Ha-ha-o-ya-da-'-te/u-so-tsu-ki-na,/a-o-i-me-wo/shi-te-i-ru-de-wa-na-i-ka $7 / 5 / 5 / 9$

Ainsi se présente visualisée la dissémination uniforme de voyelles et de consonnes. Si dans la traduction le vers japonais est deux fois plus long que l'alexandrin original, c'est à cause de l'omniprésence des voyelles. La rythmique de Nakahara a toujours pour base la cadence 7-5 : tantôt il l'inverse en 5-7, tantôt il remplace la mesure de 7 syllabes par une autre de 8 ou même 9 syllabes. Il recrée habilement en japonais le ton empathique que Rimbaud emprunte pour décrire les enfants pauvres. C'est le poète de seize ans qui représente le face-à-face tendu entre la "Mère " autoritaire et le fils apparemment docile qu'il était à sept ans. Les deux personnages ont en commun le «bleu regard", emblème de l'« hypocrisie ». Nakahara utilise une langue parlée et un ton familier, qui atténuent la rigidité de la cadence fixe.

Mais, cette traduction est parsemée d'erreurs ou d'imprécisions interprétatives : on en repère plusieurs dans l'espace des neuf vers cités. Le traducteur prend la « foire » pour un grand marché et non pour la diarrhée; l'« œil déteignant sur la joue » serait selon lui un « œil déteint »; les " pitiés immondes » signifieraient un « état pitoyable ».

\section{Renouveler les passerelles}

Hidéo Kobayashi et Chûya Nakahara ont posé les bases de la réception de Rimbaud au Japon. Longtemps, les jeunes lecteurs s'intéressant à Rimbaud ont commencé par lire leurs traductions. En 1938, huit ans après sa première publication, la Saison en enfer par Kobayashi est entrée dans la collection de poche Iwanami (Iwanami bunko); elle a fait l'objet de plus de quatre-vingts réimpressions jusqu'à aujourd'hui. Dans un sens, elle a même contribué à installer le prestige de la collection. En 2013, les Poésies de Rimbaud par Chûya Nakahara y ont fait elles aussi leur entrée.

30 À la suite de ces pionniers, plus de dix traductions de Rimbaud ont été publiées, dont quatre Euvres complètes avec correspondance générale. Au fil du temps, la précision de 
la traduction s'est progressivement améliorée. Depuis les années 1960, avec la traduction du critique Norio $A w a z u^{21}$ en particulier, Rimbaud est devenu un poète « accessible ». Les tentatives de renouvellement continuent.

31 « Des erreurs, il doit y en avoir autant que d'hydrogène dans l'eau »"22, disait Kobayashi à propos de sa traduction, comme par défi. De fait, sa traduction et celle de Nakahara déforment le sens général de l'œuvre. Pourtant, elles ont toujours séduit les lecteurs ignorants du texte original, grâce à leur intégration, fût-elle arbitraire, de la voix de Rimbaud. C'est là le problème le plus grave, et le plus agaçant, dans la réception de Rimbaud au Japon. Rien, en effet, qui sente la traduction en elles. La véhémence de la traduction de Kobayashi épouse la modulation discontinue d'Une saison en enfer; la langue lyrique, tantôt triste tantôt allègre, de Nakahara convient aux Derniers vers. Chacune de ces deux traductions devrait être davantage appréciée comme la création du traducteur que comme celle de Rimbaud ; elle relève davantage de la littérature japonaise que de la littérature française. La gloire de Rimbaud au Japon est bien singulière. Elle a été forgée sur les malentendus et à moitié usurpée. En 2013, l'éditeur Iwanami a choisi à juste titre de classer les Poésies traduites par Nakahara dans la "catégorie verte ", celle des classiques modernes de la littérature japonaise. Il devrait également déplacer dans la même catégorie Une saison en enfer par Kobayashi, qui figure depuis toujours dans la " catégorie rouge ", celle de la littérature étrangère.

Ce paradoxe lié à la renommée des deux traducteurs est resté longtemps tenace. Tous ceux qui après eux ont tenté de proposer une nouvelle traduction de Rimbaud se sont heurtés à l'adhésion inconditionnelle des lecteurs à leurs versions. Les noms de Nakahara et surtout de Kobayashi étaient devenus indissociables de celui de Rimbaud et n'ont cessé de servir de références à l'évaluation des traductions ultérieures.

Cependant, les choses ont fini par évoluer. Le vocabulaire de Kobayashi et les tournures de Nakahara semblent désormais caducs, trop éloignés du langage des jeunes lecteurs : ils ne les comprennent plus bien. D'où la triple exigence à laquelle doit répondre quiconque entreprend aujourd'hui une nouvelle traduction de Rimbaud. Celle, d'abord, de renouveler la langue de la traduction, de faire renaître Rimbaud dans une langue vivante, sans anachronisme complaisant. Puis, pour traduire son vers, celle de trouver un rythme. Les cadences toutes faites étant invalidées, la traduction du vers passe par le vers libre, qui doit «chaque fois " être capable de capter l'inner rhythm de l'auteur. Enfin celle, la plus ardue sans doute, d'avoir une compréhension préalable du texte, puisqu'il est impossible d'être un bon traducteur sans être un bon exégète ou simplement un bon lecteur. Du français au japonais, l'automatisme ne fonctionne sur aucun plan, lexical, syntaxique, culturel..., à la différence de la traduction italienne ou anglaise. Toute structure originale doit d'abord être démontée pour ensuite être reconstruite dans un édifice aussi proche que possible. Il s'agit moins d'une traduction proprement dite que d'une adaptation dirigée par l'intelligence du texte et la cohérence de l'interprétation.

Personnellement, j'ai eu deux occasions de traduire Rimbaud. La première date de 1994, quand je me suis chargée de l'ensemble des Illuminations pour les Poésies complètes ${ }^{23}$ que j'ai traduites en collaboration avec deux rimbaldiens japonais. Puis, le volume de 800 pages est passé à 1200 pages en 2006 du fait de l'ajout de la correspondance générale : il a alors été rebaptisé Cuvres complètes ${ }^{24}$. La seconde est toute récente. En 2020, j'ai réalisé une anthologie bilingue de Rimbaud ${ }^{25}$, pour la collection de poche Iwanami. Elle 
se trouve désormais dans la catégorie rouge, à côté d'Une saison en enfer par Hideo Kobayashi. C'est la première édition bilingue des œuvres de Rimbaud.

Dans les deux cas, j'ai tiré profit de modalités de présentation peu communes. Dans les Poésies complètes, 300 pages sur deux colonnes (sur deux niveaux, haut et bas, plus précisément) sont consacrées aux notes et aux commentaires, dont une centaine aux Illuminations. En intégrant le fruit des recherches rimbaldiennes menées dans le monde entier au cours des vingt années précédentes et ceux de nos lectures personnelles, nous avons voulu que la traduction soit autonome et d'accès facile, que nos notes et nos commentaires aident effectivement à la compréhension d'un texte souvent rebelle à l'interprétation. Au fond, ce volume se voulait une somme à la fois de traduction et de recherche. Mais plus d'un quart de siècle s'étant écoulé depuis les Poésies complètes, il est temps de l'augmenter ou d'envisager une nouvelle entreprise d' " Euvres complètes ", en y intégrant les textes retrouvés entre-temps (Famille maudite, Le Rêve de Bismarck) ainsi que la lettre exhumée (celle du 16 avril 1874, adressée à Jules Andrieu).

Quant à mon anthologie récente, la série où elle se trouve comptait déjà une douzaine de poètes anglo-américains, de Shakespeare à Frost en passant par Blake, Wordsworth, Keats, Poe, Whitman, Dickingson, Yeats, etc. Mais Rimbaud a été le premier poète français à y entrer. Cette anthologie bilingue, par sa structure unique, permet au lecteur une approche qui n'est pas possible dans les recueils ordinaires de poésie traduite. J'ai essayé d'en tirer le meilleur parti.

Fig. 1

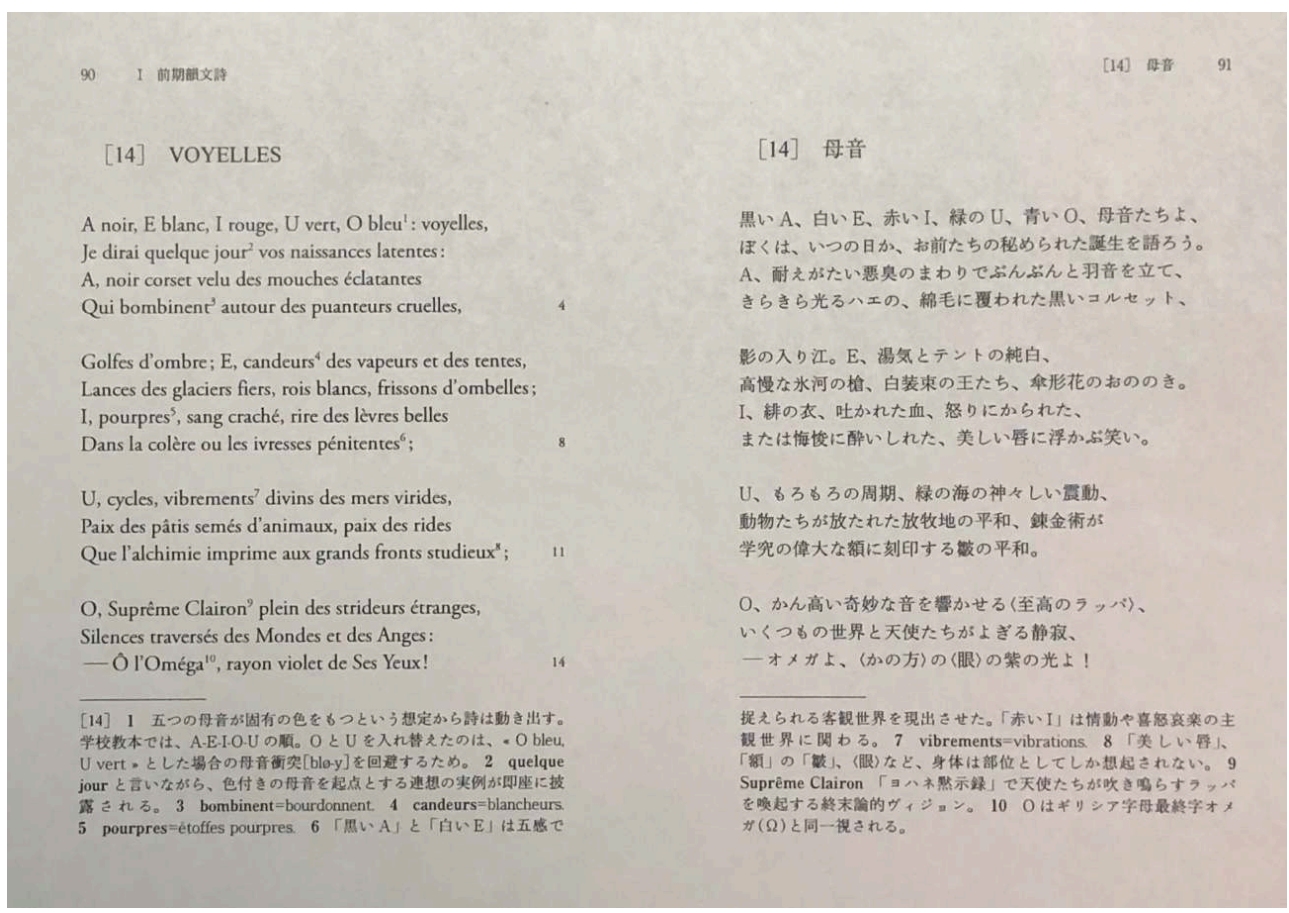

Fig. 1. Pages de Voyelles (Anthologie bilingue : Poésies de Rimbaud, 2020)

Le texte original en français sur la page de gauche et la traduction japonaise sur la page de droite, avec un espace de notes de bas de page de six lignes dans le registre inférieur, permettent de percevoir le texte de Rimbaud de trois façons en même temps. J'ai sélectionné quinze poèmes réguliers de 1870-1871, neuf parmi les Derniers vers de 1872, l'intégral d'Une saison en enfer et neuf poèmes en prose des Illuminations; en marge de 
ces quatre groupes majeurs, deux zutiques, le dizain dans l'album de Félix Régamey L'enfant qui ramassa les balles... et le Rêve inclus dans la lettre à Ernest Delahaye du 14 octobre.

Je voulais rendre ma traduction aussi fidèle et simple que possible par rapport au texte original (mais peut-être un peu plus explicative que celui-ci), afin que les lecteurs ayant une connaissance du français puissent facilement saisir la correspondance entre le texte original et la traduction japonaise. En même temps, j'ai accordé la même attention au caractère autonome de la traduction, pour que les lecteurs sans connaissance $d u$ français puissent comprendre le texte par la seule traduction japonaise, et que leur intérêt soit éveillé. Pour traduire les vers de Rimbaud, réguliers ou libérés, le seul moyen dont on dispose aujourd'hui est le vers libre. La malléabilité de celui-ci est inconditionnée, mais il exige d'épouser l'inner rythme de l'auteur. Formulée par Sakutarô Hagiwara dans les années 1920, l'idée s'impose toujours tant au traducteur qu'au poète. L'exigence d'une invention incessante s'impose également à la prose de Rimbaud. Une saison en enfer et les Illuminations ne demandent pas la même tonalité, le même parler. Pour capter une telle modulation ou inflexion, le traducteur a besoin d'un certain talent de comédien, bien qu'on sache quel danger se cache dans ce besoin comme en témoigne le cas de Hidéo Kobayashi.

Mon principe d'annotation était double : ne pas m'attacher aux endroits où le lecteur peut trouver lui-même la solution en consultant le dictionnaire, par exemple; ne pas esquiver, en revanche, les vraies difficultés que les éditions existantes ne commentent pas suffisamment voire pas du tout, comme la strophe 10 du Bateau ivre, "J'ai rêvé la nuit verte aux neiges éblouies, / [...] $\aleph^{26}$, ou l'enchainement obscur de deux phrases dans le prologue d'Une saison en enfer : «Je me suis allongé dans la boue. Je me suis séché à l'air du crime $\aleph^{27}$. Certes, les annotations destinées aux lecteurs français et celles destinées aux lecteurs japonais lisant Rimbaud à l'aide de la traduction sont nécessairement différentes, mais j'ai remarqué, depuis que j'ai commencé à lire Rimbaud, que les annotateurs passent souvent sous silence les questions auxquelles le lecteur veut vraiment obtenir une réponse. Ce silence est aussi désespérant que la question elle-même, car le lecteur se tourmente à l'idée (fausse) que lui seul est incapable de comprendre ce qui est évident pour tout le monde. Pour cette anthologie je me suis donc dit qu'il fallait au moins mentionner les difficultés là où elles se trouvent et que, même si je n'arrivais pas à les résoudre, je devais les cerner, vérifier leur nature, pour les lecteurs, « avec » les lecteurs.

Le quatrième et dernier atout de cette édition est les commentaires en fin du volume. L'édition de poche offre un espace très limité, les commentaires comme les notes doivent donc être aussi concis que possible. Dans le cas de mon anthologie, ils occupent un espace assez large, 80 sur 400 pages, mais commenter 46 poèmes en l'espace de 80 pages est un vrai exercice de concision. On ne doit dire que ce qui est essentiel, mais l'essentiel doit être dit sans omission.

41 Je puis dire que mon souhait de produire un livre bilingue qui ne laisse pas le lecteur dans l'incertitude, le genre de livre bilingue que je souhaitais autrefois quand j'ai commencé à lire Rimbaud a été réalisé. C'est comme un cadeau tardif que j'ai fait à celui que j'étais il y a près d'un demi-siècle. Mais je sais en même temps que toute traduction a une sorte de date limite de péremption. Dans trente ou quarante ans, mon anthologie semblera peut-être démodée. Une lecture plus précise et plus approfondie de Rimbaud aura peut-être émergé. À ce moment-là, la prochaine génération de traducteurs pourra 
faire de nouvelles tentatives. La poésie de Rimbaud se régénèrera avec une fraîcheur inédite dans la langue japonaise vivante de leur époque.

Fig. 2

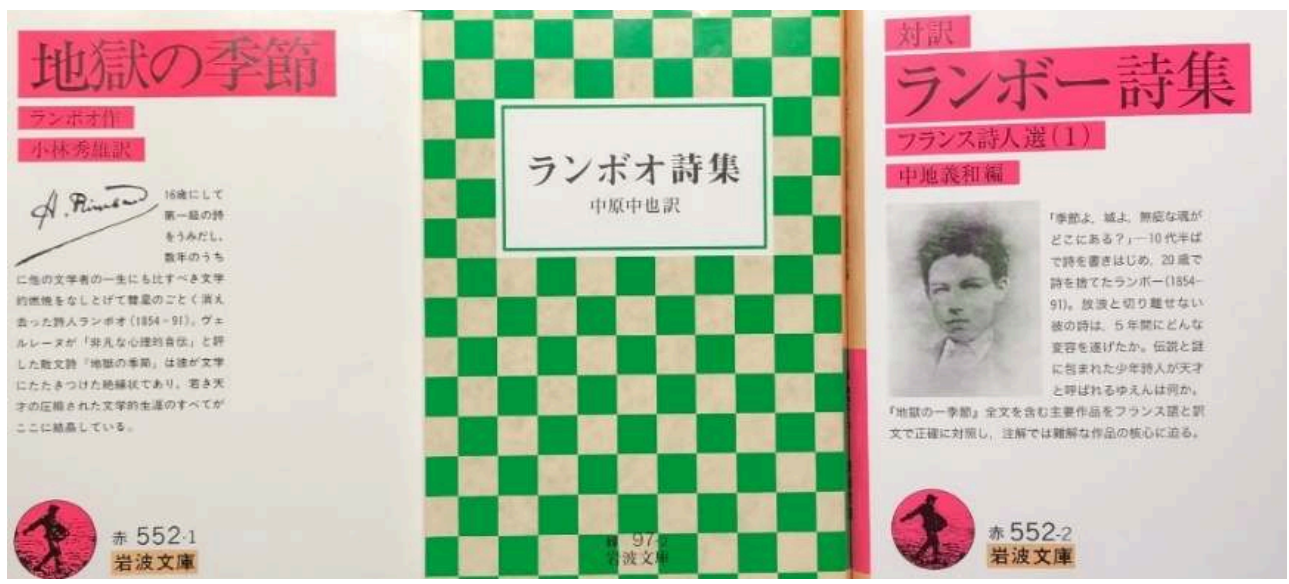

Fig. 2. Les trois volumes de Rimbaud dans la coll. «Iwanami bunko ». De gauche à droite : Une saison en enfer par Hidéo Kobayashi (catégorie rouge) ; Poésies de Rimbaud par Chûya Nakahara (catégorie verte) ; Anthologie bilingue: Poésies de Rimbaud par Yoshikazu Nakaji (catégorie rouge).

\section{NOTES}

1. M. Toyama, R. Yatabé et T. Inoué, Shintaishishô [1882], Tokyo, Kindai Bungaku kan, 1971; reproduction photographique disponible en ligne, consultée le 15/09/2021, URL: <https:// dl.ndl.go.jp/info:ndljp/pid/876377>.

2. Dans la lettre « du voyant » du 15 mai 1871 à P. Demeny : A. Rimbaud, Cuuvres complètes, éd. A. Guyaux avec la collaboration d'A. Cervoni, Paris, Gallimard, « Bibliothèque de la Pléiade », 2009, p. 346 (dorénavant OC).

3. B. Ueda, Zen yakushi-shû (Traductions poétiques complètes) [1905], Tokyo, Iwanami shoten, « Iwanami bunko », 1962 ; K. Nagai, Sango-shû [1913], Tokyo, Iwanami shoten, « Iwanami bunko », 1991 ; D. Horiguchi, Gekka no ichigun [1925], Tokyo, Kôdansha, « Kôdansha bunko », 1996.

4. B. Ueda publiera sa traduction des Chercheuses de poux dans Joshi bundan (Scène littéraire féminine) du $1^{\mathrm{er}}$ mai 1909. C'était la toute première traduction japonaise de Rimbaud.

5. D. Horiguchi, « Postface du traducteur » dans Id., Gekka no ichigun [1925], Tokyo, Hakusuisha, 1957 ; repris dans Id., Gekka no ichigun, cit., 1996, p. 599-600.

6. Ibid., p. 603.

7. Les trois essais sont réunis dans H. Kobayashi, Zenshû (Euvres complètes), Tokyo, Shinchôsha, 1968, t. II, p. 135-177.

8. Id., « Rimbaud I », dans Id., CEuvres complètes, cit., t. II, p. 140-141.

9. Paul Valéry parle d'« un son grave et continu», de «cette profonde note de l'existence », P. Valéry, « Note et digression », dans Id., CEuvres I, Paris, Gallimard, « Bibliothèque de la Pléiade », 1957, p. 1228.

10. H. Kobayashi, «Ashiru to kamé no ko » (Achille et la petite tortue), dans Id., CEuvres complètes, cit., 1967, t. I, p. 46.

11. Id., Euvres complètes, cit., t. II, p. 152. 
12. OC, p. 245.

13. On peut évoquer "Je veux devenir bien fou de rage" (A. Rimbaud, Vierge folle, dans $O C$, p. 260); l'«hallucination simple» et "l'hallucination des mots", le "désordre de mon esprit », les "sophismes de la folie ", les «enchantements assemblés sur mon cerveau » (Id., Alchimie du verbe, dans $O C$, p. 265 et 268 ), ainsi que le «dérèglement de tous les sens » dans les deux lettres « du voyant » de mai 1871 (OC, p. 340 et 344 ).

14. Ibid., p. 260.

15. Ibid., p. 268.

16. Ibid., p. 217-218

17. De 1942 à 1950, les jeunes écrivains francisants du cercle de la « Matinée poétique ", Shûichi Katô, Takahiko Fukunaga, Shin'ichirô Nakamura, entre autres, se réunissaient régulièrement pour réciter entre eux leurs créations poétiques en vers rimés. Ils publient en 1948 le Recueil de poèmes de la « Matinée poétique». Mais, vivement critiqués de l'inanité de leurs entreprises par le grand poète Tatsuji Miyoshi, ils les abandonnent peu de temps après.

18. S. Hagiwara, Zenshû (Euvres complètes), Tokyo, Tchikuma shobô, 1975, t. I, p. 245.

19. OC, p. 126.

20. A. Rimbaud, Rambô shishû (Poésies de Rimbaud), tr. Ch. Nakahara, commentaire de H. Usami, Tokyo, Iwanami shoten, «Iwanami bunko», 2013, p.73-74. Dans l'écriture japonaise sont mélangés les caractères chinois qui sont idéographiques, les hiragana qui sont des caractères phonétiques et les katakana qui sont également des caractères phonétique mais destinés spécifiquement aux mots d'origine étrangère, occidentale en particulier.

21. Id., Poésies complètes, tr. Norio Awazu, Tokyo, Shichôsha, 1965.

22. H. Kobayashi, « Postface » à la première édition de sa traduction d'Une saison en enfer, Tokyo, Hakusuisha, 1930.

23. A. Rimbaud, Poésies complètes, tr., notes et notice par H. Hirai, H. Yuasa et Y. Nakaji, Tokyo, Seidosha, 1994.

24. Id., Euvres complètes, traduction, notes et notice par H. Hirai, H. Yuasa, Y. Nakaji et Y. Kawanabé, Tokyo, Seidosha, 2006

25. Anthologie bilingue : Poésies de Rimbaud, tr., notes et commentaires par Y. Nakaji, Tokyo, Iwanami shoten, «Iwanami bunko », 2020.

26. OC, p. 163.

27. Ibid., p. 245.

\section{RÉSUMÉS}

C'est dans les années 1930, grâce à la traduction d'Une saison en enfer par le critique Hideo Kobayashi et des Poésies par le poète Chûya Nakahara, que Rimbaud commence à s'ancrer dans la culture japonaise comme l'un des poètes étrangers les plus prestigieux. Les traductions de personnalités célèbres ont favorisé la réception de Rimbaud, mais il est tout aussi vrai que leur renommée a été renforcée par le fait qu'ils étaient les traducteurs de Rimbaud. Cependant, leurs traductions révèlent des problèmes linguistiques à tel point qu'on pourrait les qualifier de vitrine de mauvaises traductions. La gloire du poète, comme celle des traducteurs engendrée par la traduction est une gloire singulière basée sur le malentendu. La véritable compréhension de Rimbaud ne commencera que lorsque, la mauvaise empreinte de ces traductions étant éradiquée, 
un nouveau traducteur fournira une traduction linguistiquement exacte et appropriée à la langue de l'époque.

It is in the 1930s, thanks to the translation of A Season in Hell by the critic Hideo Kobayashi and the Poems by the poet Chûya Nakahara, that Rimbaud begins to be anchored in the Japanese culture as one of the most prestigious foreign poets. The translations of famous people helped Rimbaud's reception, but it is also true that their fame was enhanced by the fact that they were Rimbaud's translators. However, their translations reveal linguistic problems to such an extent that they could be described as a showcase of bad translations. The glory of the poet as well as that of the translators generated by the translation is a singular glory based on misunderstanding. The true understanding of Rimbaud would only begin when, with the bad imprint of these translations eradicated, a new translator would provide a linguistically accurate and appropriate translation to the language of the time.

INDEX

Mots-clés : Rimbaud (Arthur), Japon, traduction, réception, gloire Keywords : Rimbaud (Arthur), Japan, translation, reception, glory 